\title{
Loss of heterozygosity: what is it good for?
}

Georgina L. Ryland ${ }^{1,2}$, Maria A. Doyle ${ }^{3}$, David Goode ${ }^{4}$, Samantha E. Boyle ${ }^{1}$, David Y.H. Choong 1 , Simone M. Rowley ${ }^{1}$, Jason Li ${ }^{3}$, Australian Ovarian Cancer Study Group ${ }^{6}$, David DL Bowtell ${ }^{7}$, Richard W. Tothill ${ }^{8}$, lan G. Campbell ${ }^{1,5,9}$ and Kylie L. Gorringe $e^{1,5,9^{*}}$

\begin{abstract}
Background: Loss of heterozygosity $(\mathrm{LOH})$ is a common genetic event in cancer development, and is known to be involved in the somatic loss of wild-type alleles in many inherited cancer syndromes. The wider involvement of $\mathrm{LOH}$ in cancer is assumed to relate to unmasking a somatically mutated tumour suppressor gene through loss of the wild type allele.
\end{abstract}

Methods: We analysed 86 ovarian carcinomas for mutations in 980 genes selected on the basis of their location in common regions of $\mathrm{LOH}$.

Results: We identified 36 significantly mutated genes, but these could only partly account for the quanta of LOH in the samples. Using our own and TCGA data we then evaluated five possible models to explain the selection for non-random accumulation of $\mathrm{LOH}$ in ovarian cancer genomes: 1. Classic two-hit hypothesis: high frequency biallelic genetic inactivation of tumour suppressor genes. 2. Epigenetic two-hit hypothesis: biallelic inactivation through methylation and LOH. 3. Multiple alternate-gene biallelic inactivation: low frequency gene disruption. 4. Haplo-insufficiency: Single copy gene disruption. 5. Modified two-hit hypothesis: reduction to homozygosity of low penetrance germline predisposition alleles. We determined that while high-frequency biallelic gene inactivation under model 1 is rare, regions of $\mathrm{LOH}$ (particularly copy-number neutral LOH) are enriched for deleterious mutations and increased promoter methylation, while copy-number loss LOH regions are likely to contain under-expressed genes suggestive of haploinsufficiency. Reduction to homozygosity of cancer predisposition SNPS may also play a minor role.

Conclusion: It is likely that selection for regions of $\mathrm{LOH}$ depends on its effect on multiple genes. Selection for copy number neutral LOH may better fit the classic two-hit model whereas selection for copy number loss may be attributed to its effect on multi-gene haploinsufficiency. $\mathrm{LOH}$ mapping alone is unlikely to be successful in identifying novel tumour suppressor genes; a combined approach may be more effective.

Keywords: Tumour suppressor gene, SNP, Ovarian cancer, Mutation, Haploinsufficiency

\section{Background}

Cancer cells undergo multiple genetic and epigenetic hits in the development of tumorigenic phenotypes, including somatic point mutations, increases in copy number, gene deletions, gene rearrangements, translocations and promoter hypermethylation [1]. These random events are selected for due to their effect on oncogenes, where the aberration activates the gene to promote

\footnotetext{
*Correspondence: kylie.gorringe@petermac.org

${ }^{1}$ Cancer Genetics Laboratory, Peter MacCallum Cancer Centre, East

Melbourne, Victoria, Australia

${ }^{5}$ Sir Peter MacCallum Department of Oncology, University of Melbourne,

Parkville, Victoria, Australia

Full list of author information is available at the end of the article
}

tumorigenesis (e.g. KRAS, $M Y C$ ), and on tumour suppressor genes (TSG), where the genetic or epigenetic aberrations is inactivating (e.g. TP53, PTEN), since the normal function of these genes is to restrict tumorigenic potential.

Loss of heterozygosity $(\mathrm{LOH})$ is a common genetic event in many cancer types, so-called because of the early observations of a change in polymorphic markers from a heterozygous state in the germline to an apparently homozygous state in the tumour DNA [2]. LOH is a general term that encompasses both $\mathrm{LOH}$ with copy number losses (CNL-LOH) and copy number neutral $\mathrm{LOH}$ (CNN-LOH). In CNL-LOH all or part of a chromosome is deleted. $\mathrm{CNN}-\mathrm{LOH}$ originates either 
through a homologous recombination event ("gene conversion"), or because the retained chromosome was duplicated either before or after the $\mathrm{LOH}$ event. $\mathrm{LOH}$ is strongly associated with loss of the wild-type allele in individuals with an inherited cancer predisposition syndrome and carry a germline mutation in genes such as $R B 1$ in retinoblastoma or BRCA1 in breast and ovarian cancer $[2,3]$. This "second hit" hypothesis was initially proposed by Knudson based on his observations of the incidence of familial retinoblastoma [4] and has been widely accepted as a mechanism for the complete inactivation of tumour suppressor genes, both in a germline context and the sporadic cancer context where the first hit is a somatic event, such as mutation of TP53. As a consequence, mapping of common regions of minimal LOH has historically been a popular strategy to pursue the identification of novel TSGs without the need for segregation data from large cancer families. However, such analyses have been generally been unsuccessful leading to speculation that the approach is technically and conceptually flawed [5], and even to whether there is any selective advantage to $\mathrm{LOH}$ events. Nonetheless, we previously used SNP mapping arrays to analyse $\mathrm{LOH}$ in ovarian carcinomas of diverse histological subtypes, with the rationale that the newer methodology would at least overcome some of the previous technical issues with $\mathrm{LOH}$ analyses [6]. We mapped a number of minimal regions of $\mathrm{LOH}$ containing tumour suppressor gene candidates, including regions of homozygous deletion encompassing genes such as MAP2K4 [7]. Advances in massively parallel sequencing has enabled the current study where we report targeted sequencing of 980 candidate tumour suppressor genes in 86 ovarian carcinomas, most of which have matched SNP array data enabling the assessment of the importance of $\mathrm{LOH}$ in the selection for somatic mutations in ovarian cancer. We evaluated a number of different histological subtypes, since these have different etiologies and causative genes.

\section{Methods}

\section{Ethics statement}

Accrual and use of patient material for this study was approved by the following Human Research Ethics Committees: Peter MacCallum Cancer Centre Human Research Ethics Committee, Southampton Hospital Human Research Ethics Committee, University of Melbourne Human Research Ethics Committee, Queensland Institute of Medical Research Human Research Ethics Committee, Westmead Hospital Human Research Ethics Committee. All individuals gave written informed consent for the use of their tissue in research. This project was approved by the Peter MacCallum Cancer Centre Human Research Ethics Committee (Approval \# 09/29).

\section{Ovarian tumour cohort}

A tumour cohort $(\mathrm{n}=86)$ comprising a variety of histological subtypes including serous $(\mathrm{n}=45)$, endometrioid $(n=28)$, mucinous $(n=7)$ and clear cell $(n=6)$ were obtained through the Australia Ovarian Cancer Study, the Peter MacCallum Cancer Centre Tissue Bank, or from patients presenting to hospitals in the south of England [8]. The majority of tumour DNA samples were needle microdissected to ensure greater than $70 \%$ cancer epithelial cell component; other samples were processed from tissue where the reference haematoxylin and eosin stained section showed $>70 \%$ tumour epithelial cells. Matching peripheral blood samples were also collected from patients at time of tumour collection and used as a source of germline DNA for somatic mutation detection. Details of the cohort are listed in Additional file 1: Table S1.

\section{Library preparation, target enrichment and sequencing}

Library preparation was performed as previously described [9] following the Illumina genomic DNA library preparation protocol (Illumina, San Diego, CA) using an input of $200 \mathrm{ng}$ of tumour or matched normal lymphocyte DNA. Seven custom multiplexing adapters compatible with Illumina single-end sequencing were used and indexed DNA samples were pooled equally prior to PCR enrichment. A boutique exon capture (SureSelect, Agilent Technologies, Santa Clara, CA) was used to enrich for coding exons of candidate tumour suppressor genes $(\mathrm{n}=980$, Additional file 1: Tables S2 and S3) and known cancer genes (TP53, $B R C A 1, B R C A 2)$ according to the recommended protocol. Capture probes were designed using default parameters in eArray (Agilent Technologies).

Sequencing of target-enriched DNA libraries were performed using an Illumina GAIIx, generating 75 bp single-end sequence reads. Image analysis and base calling was performed using the Genome Analyser Pipeline v1.5-1.7. Sequence reads were aligned to the human reference genome (GRCh37/hg19 assembly) using BWA [10] and any remaining unmapped reads aligned with Novoalign [11]. The mean coverage for bases within target regions was 70 -fold and $92 \%$ had at least 10 -fold coverage. This was followed by local realignment with GATK [12]. Point mutations and insertions/deletions (indels) were identified using GATK and Dindel [13] respectively, and annotated according to Ensembl release 56. Sequence variants were called as somatic alterations only when $(i)$ the variant was not called in the matched normal sample or identified as a germline alteration in another tumour/normal pair (ii) the variant was not seen in $>=2$ independent reads in the matched normal sample following manual inspection of sequence reads using the Integrated Genomics Viewer [14] (iii) the variant was identified in bi-directional sequence reads. 
A selection of variants that met the above criteria for a somatic mutation $(n=202)$ were subjected to validation by conventional PCR amplification and bidirectional capillary electrophoresis on the ABI3130 Genetic Analyser using BigDye Terminator v3.1 sequencing chemistry (Applied Biosystems, Foster City, CA).

\section{SNP arrays and loss of heterozygosity}

Affymetrix SNP Mapping array data was obtained for the 86 sequenced cases, 54 by SNP6 arrays (GSE19539, [15]), 26 by $500 \mathrm{~K}$ arrays (previously published in [6]), and six previously unreported low-grade endometrioid cases. Affymetrix SNP6 CEL files, HM27 methylation array data (level 3), Agilent expression array data (level 3) and somatic mutation data from 266 tumors generated by The Cancer Genome Atlas (TCGA) were downloaded from the TCGA Data Portal. LOH was detected as described previously in Partek Genomics Suite (Partek, St Louis, MO), using allele-specific copy number that compared the tumour genotype to the matching normal genotype, and evaluated the copy number at heterozygous alleles [6]. The "min" allele had to have a value of $<0.5$ copies to be called $\mathrm{LOH}$, thus excluding regions of allelic imbalance where at least one copy of both alleles was present. The results published here are in whole or part based upon data generated by The Cancer Genome Atlas pilot project established by the NCI and NHGRI. Information about TCGA and the investigators and institutions who constitute the TCGA research network can be found at http://cancergenome.nih.gov.

\section{Results and discussion}

\section{A candidate TSG screen in ovarian cancer - selection of} genes from $\mathrm{LOH}$ regions

Candidate ovarian tumour suppressor genes $(n=980)$ were selected for analysis on the basis of their location in frequent regions of $\mathrm{LOH}$ or deletion (Additional file 1: Tables S2 and S3) from our previously published SNP array analysis of 122 primary ovarian carcinomas of various histologies [6]. The regions, located on 20 different chromosome arms, met the following three criteria. Firstly, minimal overlapping regions of $\mathrm{LOH}$ were included if they were detected in greater than $35 \%$ of all ovarian carcinomas analysed, or secondly, in $>35 \%$ of subtype specific minimal overlapping regions of $\mathrm{LOH}$ ( 4 of 9 clear cell carcinomas, 5 of 12 low-grade endometrioid carcinomas (grades 1 and 2), 6 of 16 mucinous carcinomas and 23 of 64 high-grade serous/endometrioid carcinomas (grades 2 and 3 for the serous subtype, grade 3 for endometrioid carcinomas)). Finally, all homozygous deletions within frequent regions of $\mathrm{LOH}$ along with the overlapping portion of all recurrent homozygous deletions were included. This gene list included genes with well established roles in cancer such as CDKN2A and PTEN, but for the purposes of this analysis they were included in the "candidate" LOH genes. In addition, the known ovarian cancer genes TP53, BRCA1 and BRCA2 were included despite lying outside the minimal regions of $\mathrm{LOH}$.

\section{A candidate TSG screen in ovarian cancer - correlation of mutations with LOH}

A targeted mutation screen was conducted on the 86 ovarian cancer cases including high-grade serous and endometrioid, low-grade endometrioid, clear cell and mucinous subtypes. Somatic coding mutations were detected in both candidate (561 variants in 366 genes, Additional file 2: Table S4) and known cancer genes (58 TP53 mutations in 56 cases and two mutations in $B R C A 1)$. Eighty-nine genes had two or more nonsynonymous mutations. The classic two-hit hypothesis would predict that driver genes have homozygous, deleterious mutations in samples with $\mathrm{LOH}$. With respect to deleterious mutation status this was certainly true for TP53 and BRCA1 where a high proportion of somatic mutations were truncating (25/58 and $2 / 2$, respectively) compared to an overall truncating mutation frequency of $13 \%$ (72/561). In addition, among the 53 cases with TP53 or BRCA1 somatic mutations where SNP data was available, 50 (94 \%) showed LOH of the wild-type allele. This was is sharp contrast with the other candidate genes, where only 181/ 520 showed LOH of the wild-type allele (35\%); in particular, there was no significant difference in $\mathrm{LOH}$ of the wild-type allele between non-synonymous mutations (134/381 with LOH, $35.2 \%)$ and synonymous mutations (47/139 with LOH, $33.8 \%$ ). The overall frequency of non-synonymous compared to synonymous mutations was $73 \%(411 / 561)$ for the candidate TSGs, but $100 \%$ of mutations in known cancer genes were non-synonymous (60/60). This difference in ratio suggests that the majority of mutations in candidate TSGs from LOH regions are likely to be passenger events, since this rate might be expected without any strong positive selection [16]. The lack of difference in $\mathrm{LOH}$ between synonymous and non-synonymous also implies that there is limited selection for homozygosity for the majority of gene mutations.

\section{Significance analysis of recurrently mutated gene candidates}

Within the list of mutated genes, we applied a number of filters to assess whether any genes could function as tumor suppressors under either a one-hit or two-hit mechanism. Firstly, significantly mutated genes were identified using the $\mathrm{MuSiC}$ algorithm [17], which determines the significance of the observed mutation rate of each gene based on the background mutation rate in the sample cohort. Three known ovarian cancer genes (TP53, 
PTEN and CDKN2A) were identified by all three tests (convolution, likelihood ratio and Fisher's combined pvalue tests) with a false discovery rate (FDR) of less than 0.10. At this FDR the genes DNAH9, LINGO1, MEF2C, SAMD11, STARD5, ZNRF4 and ZNF287 were also identified, although each was supported only by the likelihood ratio test.

Secondly, the 125 genes with recurrent mutations were assessed for the proportion of cases with biallelic mutation, including by homozygous deletion from SNP array data. For genes with mutations in three or more cases, the proportion of biallelic mutations was greater than $80 \%$ of mutated samples for eight genes (AL355987.1, CASK, CDKN2A, MAP2K4, NF1, PTEN, RB1 and TP53) and between 60-80 \% for seven genes (FANCA, GRAMD4, GPR98, IL16, MYOCD, SYNE1 and TEX15). Biallelic mutations were detected in $2 / 2$ mutated samples in an additional nine genes (SKG223, APOOL, BRCA1, CDH8, DACH2, EPHX2, FARP1, PNMA3 and RAI1).

Finally, genes recurrently targeted by inactivating mutations were identified. Mutations with overtly deleterious consequences were considered for this analysis, including nonsense and essential splice site mutations, frameshift indels and gene deletions. Although missense amino acid changes and in-frame indels can also negatively impact gene function, interpreting these mutations in the absence of functional validation is challenging. Sixteen genes were identified where more than half of their mutations would be considered clearly deleterious, including seven known ovarian cancer genes (PTEN, CDKN2A, MAP2K4, PIK3R1, RB1, FANCA and BRCA1). These three analyses identified 36 genes as possible tumour suppressors (Table 1), and it was notable that seven well characterised tumour suppressors were identified by at least two of the three methods (BRCA1, TP53, RB1, PTEN, CDKN2A, FANCA, and MAP2K4), although others were only identified by one method (NF1, PIK3R1). In contrast, 22 of the 27 (81\%) novel/less well characterized genes were identified by only 1 method, indicating that regions of $\mathrm{LOH}$ are not strongly enriching for novel genes with classic tumor suppressor gene characteristics.

\section{Loss of heterozygosity - what is it good for?}

From the data above it appears that we did not identify dominant, very frequently mutated novel genes where selection for a classic two-hit tumour suppressor gene was apparent. So what, if anything, is the $\mathrm{LOH}$ for? We considered five possibilities (Fig. 1) and assessed each in turn.

1. Classic two-hit hypothesis: high frequency biallelic genetic inactivation of TSG
2. Epigenetic two-hit hypothesis: biallelic inactivation through methylation and $\mathrm{LOH}$

3. Multiple alternate gene biallelic inactivation: low frequency gene disruption

4. Haplo-insufficiency: Single copy gene disruption

5. Modified two-hit hypothesis: reduction to homozygosity of predisposition alleles

1. Classic two-hit hypothesis: high frequency biallelic genetic inactivation of TSG

This mechanism is demonstrably true for many known tumour suppressor genes, with TP53 being a clear example of a gene functioning as a classical TSG in ovarian cancer [18, 19]. However, from our data and large published studies such as TCGA, it is clear that novel genes with a high frequency of biallelic mutations are exceedingly rare and can not explain the bulk of the observed $\mathrm{LOH}$. For example, $8 \mathrm{p}$ undergoes $\mathrm{LOH}$ in $>40 \%$ of ovarian carcinomas, but no gene in this region is mutated at frequency higher than $3 \%$ in our or any other study, although homozygous deletion can target, for example, CSMD1 in $11 \%$ of cases [20]. It remains a possibility, however, that genes not represented on our targeted or exome sequencing platforms could still be the target of such $\mathrm{LOH}$, for example long non-coding RNAs.

2. Epigenetic two-hit hypothesis: high frequency biallelic inactivation through methylation and $\mathrm{LOH}$

Somatic gene mutation is not the only mechanism of biallelic inactivation. Some TSGs can be inactivated through a combination of $\mathrm{LOH}$ and promoter hypermethylation, for example $M L H 1$. This methylation can be acquired somatically or may be a consequence of imprinting. We assessed this possibility using TCGA ovarian cancer methylation data. Globally, we observed that there was no enrichment for methylation in regions of $\mathrm{LOH}$ - in samples with $\mathrm{LOH}$ at a locus, on average $12.7 \%$ of genes were strongly methylated (probe value of $>0.75$ ), whereas $13.65 \%$ of genes were strongly methylated when there was no LOH, (Fig. 2a). CNLLOH was less likely to have strongly methylated genes than CNN-LOH (12.3\% vs $12.9 \%, \mathrm{p}<0.0001$, Chisquared test). However, when we analysed the $\mathrm{X}$ chromosome separately, we found that samples with any $\mathrm{LOH}$ were more likely to have low methylation levels ( $45.3 \%$ of genes had a probe value of $<0.25$, compared to $35.5 \%$ in samples without LOH, p $<0.0001$ Chi-squared test).

Detection of methylation is challenging from both technical and biological perspectives. Tumour and cell 
Table 1 Selected mutated genes in candidate TSG screen

\begin{tabular}{|c|c|c|c|c|c|c|}
\hline Gene & Location & Description & Recessive TSG $^{a}$ & Predominant subtypes & TCGA mutated ${ }^{\mathrm{b}}$ & Detected by \\
\hline$\overline{A N K R D 32}$ & $5 q 15$ & ankyrin repeat domain 32 & & $\mathrm{HG} S / \mathrm{E}+\mathrm{LGE}+\mathrm{CC}$ & 9 & Deleterious \\
\hline APOOL & Xq21.1 & apolipoprotein O-like & & HG S/E & 0 & Biallelic \\
\hline$B R C A 1$ & $17 q 21.31$ & breast cancer 1 , early onset & Y & $\mathrm{HG} S / \mathrm{E}$ & 12 & Biallelic, Deleterious \\
\hline C9ORF172 & $9 q 34.3$ & $\begin{array}{l}\text { chromosome } 9 \text { open reading } \\
\text { frame } 172\end{array}$ & & $\mathrm{HG} S / \mathrm{E}$ & 5 & Biallelic \\
\hline CACNAIB & $9 q 34$ & $\begin{array}{l}\text { calcium channel, voltage-dependent, } \\
N \text { type, alpha } 1 \text { B subunit }\end{array}$ & & $L G E+C C$ & 7 & Deleterious \\
\hline CASK & Xp11.4 & $\begin{array}{l}\text { calcium/calmodulin-dependent } \\
\text { serine protein kinase }\end{array}$ & & HG S/E & 2 & Biallelic \\
\hline $\mathrm{CDH} 8$ & $16 q 22.1$ & cadherin 8 , type 2 & & HG S/E & 9 & Biallelic \\
\hline CDKN2A & $9 p 21$ & $\begin{array}{l}\text { cyclin-dependent kinase } \\
\text { inhibitor } 2 \mathrm{~A}\end{array}$ & Y & Muc & 8 & MuSiC, Biallelic, Deleterious \\
\hline CYLCI & Xq21.1 & $\begin{array}{l}\text { cylicin, basic protein of sperm } \\
\text { head cytoskeleton } 1\end{array}$ & & $\mathrm{HG}$ S/E & 4 & Deleterious \\
\hline $\mathrm{DACH} 2$ & Xq21.3 & $\begin{array}{l}\text { dachshund homolog } 2 \\
\text { (Drosophila) }\end{array}$ & & HG S/E & 3 & Biallelic \\
\hline DNAH9 & $17 p 12$ & dynein, axonemal, heavy chain 9 & & LGE & 16 & MuSiC \\
\hline EPHX2 & $8 p 21$ & epoxide hydrolase 2, cytoplasmic & & $\mathrm{HG} S / \mathrm{E}$ & 12 & Biallelic \\
\hline FANCA & $16 q 24.3$ & $\begin{array}{l}\text { Fanconi anemia, complementation } \\
\text { group A }\end{array}$ & Y & $\mathrm{HG} S / \mathrm{E}$ & 12 & Biallelic, Deleterious \\
\hline FARP1 & $13 q 32.2$ & $\begin{array}{l}\text { FERM, RhoGEF (ARHGEF) and } \\
\text { pleckstrin domain protein } 1\end{array}$ & & HG S/E & 3 & Biallelic \\
\hline GPR98 & $5 q 13$ & G protein-coupled receptor 98 & & $\mathrm{HG}$ S/E & 17 & Biallelic \\
\hline GRAMD4 & $22 q 13.31$ & GRAM domain containing 4 & & HG S/E & 18 & Biallelic, Deleterious \\
\hline IL16 & $15 q 26.3$ & interleukin 16 & & $\mathrm{HG}$ S/E & 4 & Biallelic, Deleterious \\
\hline LINGO1 & $15 q 24.3$ & $\begin{array}{l}\text { leucine rich repeat and Ig } \\
\text { domain containing } 1\end{array}$ & & HG S/E & 4 & MuSiC \\
\hline MAP2K4 & $17 p 12$ & $\begin{array}{l}\text { mitogen-activated protein } \\
\text { kinase kinase } 4\end{array}$ & Y & $\mathrm{HG}$ S/E & 12 & Biallelic, Deleterious \\
\hline MEF2C & $5 q 14.3$ & myocyte enhancer factor $2 \mathrm{C}$ & & $\mathrm{HG}$ S/E & 8 & MuSiC \\
\hline MYOCD & $17 p 11.2$ & myocardin & & HG S/E & 7 & Biallelic, Deleterious \\
\hline NF1 & $17 q 11.2$ & neurofibromin 1 & Y & HG S/E & 37 & Biallelic \\
\hline PIK3R1 & $5 q 13.1$ & $\begin{array}{l}\text { phosphoinositide-3-kinase, } \\
\text { regulatory subunit } 1 \text { (alpha) }\end{array}$ & Y & LGE & 6 & Deleterious \\
\hline PNMA3 & $X q 28$ & paraneoplastic Ma antigen 3 & & $\mathrm{HG}$ S/E & 2 & Biallelic \\
\hline PTEN & $10 q 23$ & $\begin{array}{l}\text { phosphatase and tensin } \\
\text { homolog }\end{array}$ & Y & LGE & 25 & MuSiC, Biallelic, Deleterious \\
\hline RAl1 & $17 p 11.2$ & retinoic acid induced 1 & & $\mathrm{HG}$ S/E & 4 & Biallelic \\
\hline$R B 1$ & $13 q 14.2$ & retinoblastoma 1 & Y & $\mathrm{HG} S / \mathrm{E}$ & 32 & Biallelic, Deleterious \\
\hline RPS6KA6 & Xq21.1 & $\begin{array}{l}\text { ribosomal protein } 56 \text { kinase, } \\
90 \mathrm{kDa} \text {, polypeptide } 6\end{array}$ & & $H G S / E+L G E$ & 0 & Deleterious \\
\hline SAMD11 & $1 p 36.33$ & $\begin{array}{l}\text { sterile alpha motif domain } \\
\text { containing } 11\end{array}$ & & HG S/E & 7 & MuSiC, Deleterious \\
\hline SKG223 & $8 p 23.1$ & Sugen kinase 223 & & HG S/E + Muc & 7 & Biallelic \\
\hline STARD5 & $15 q 26$ & $\begin{array}{l}\text { StAR-related lipid transfer } \\
\text { (START) domain containing } 5\end{array}$ & & $\mathrm{HG} S / \mathrm{E}$ & 1 & MuSiC \\
\hline SYNE1 & $6 q 25$ & $\begin{array}{l}\text { spectrin repeat containing, } \\
\text { nuclear envelope } 1\end{array}$ & & $\mathrm{HG} S / \mathrm{E}$ & 14 & Biallelic \\
\hline
\end{tabular}


Table 1 Selected mutated genes in candidate TSG screen (Continued)

\begin{tabular}{|c|c|c|c|c|c|c|}
\hline TEX15 & $8 p 22$ & testis expressed 15 & & HG S/E & 13 & Biallelic \\
\hline TP53 & 17p13.1 & tumor protein p53 & Y & HG S/E & 302 & MuSiC, Biallelic \\
\hline ZNF287 & 17p11.2 & zinc finger protein 287 & & HG S/E & 4 & MuSiC, Deleterious \\
\hline ZNRF4 & $19 p 13.3$ & zinc and ring finger 4 & & HG S/E & 1 & MuSiC \\
\hline
\end{tabular}

LG E, low-grade endometrioid; HG S/E, high-grade serous/endometrioid; Muc, mucinous; CC, clear cell

${ }^{a}$ Known recessive tumour suppressor gene according to the Cancer Gene Census [32]

${ }^{b}$ Number of high-grade serous TCGA samples with somatic point mutations and indels including large homozygous deletions. Mutation data for 316 TCGA samples [33] was accessed through the cBio Cancer Genomic Portal [34]

type heterogeneity may influence the degree of methylation detected, so we also took an alternative approach where we used the methylation array data to test whether there were genes that were more strongly methylated in samples with LOH compared to samples without $\mathrm{LOH}$. Using a multiple testing correction p-value threshold of $2.2 \times 10^{-6}$, there were $1584 / 22374$ (7 \%) methylation probes that were significantly differentially methylated. Interestingly, $28 \%$ of these significant probes were located on the $\mathrm{X}$ chromosome and indeed $51 \%$ of all probes on the $\mathrm{X}$ chromosome were significantly differentially methylated, with lower average levels of methylation in samples with $\mathrm{LOH}$ compared to samples without $\mathrm{LOH}$. On the autosomes, the outcome was reversed: $50.3 \%$ of the statistically significant probes had a fold-change difference in mean methylation of $>1.5$, while only $1.1 \%$ had a fold-change difference of $<0.75$ (Fig. 2b). Thus, for the $\mathrm{X}$ chromosome it appears there is selection for retaining the active copy, perhaps because loss of this copy would be cell lethal as an effective homozygous inactivation of the chromosome. In contrast, for the autosomes there appears to be selection for increased methylation by $\mathrm{LOH}$.

We also evaluated whether there was any difference by the type of $\mathrm{LOH}$. For those genes occurring in a region of $\mathrm{LOH}$ with at least $20 \%$ frequency, we determined whether a probe was in a CNL-LOH enriched locus ( $>66 \%$ of samples with $\mathrm{LOH}$ also had $\mathrm{CN}$ loss) or a CNN-LOH enriched locus ( $>66 \%$ of samples with $\mathrm{LOH}$ were $\mathrm{CNN}$ ). Of the CNL-enriched probes, $11.3 \%$ were significantly differentially methylated, compared to $21.7 \%$ of $\mathrm{CNN}$-enriched probes ( $\mathrm{p}<0.0001$, Chi-squared test, Fig. 2c). This data would support a model whereby differential methylation is more commonly selected for in regions of CNN-LOH than CNL-LOH.

3. Multiple alternate gene biallelic inactivation: low frequency gene disruption

Another possibility is that particular loci harbour multiple TSGs but individual tumours only require one to be inactivated and the gene targeted can differ from tumour to tumour. If this is the case then locating the
TSGs by mapping overlapping regions of $\mathrm{LOH}$ would incorrectly flag the interval between two TSG as the likely location of the TSG - in effect then the peak LOH regions may not be the most likely places to find the targeted gene(s). To evaluate this possibility, we used TCGA data to see whether regions of $\mathrm{LOH}$ were enriched for somatic mutations on a sample-by-sample basis. Cases with both somatic exome and SNP array data were used $(n=266)$. There were 13,148 coding somatic mutations, of which $29.7 \%$ were located within a region of $\mathrm{LOH}$ in the sample where it was observed. The average overlap of all the genes assayed with regions of $\mathrm{LOH}$ per sample was $35.5 \%$. Thus, somatic mutations are if anything under-represented in regions of $\mathrm{LOH}$ (Binomial test $\mathrm{p}<0.0001$ ). Given that most of these mutations are likely to be passengers, we evaluated whether this was true for non-synonymous or overtly deleterious mutations (nonsense, frameshift, essential splice site). For deleterious mutations, $38.2 \%$ were in regions of LOH $(\mathrm{p}=0.035$, Binomial test), whereas only $25.2 \%$ of the non-synonymous mutations were in regions of $\mathrm{LOH}$, similar to the $22.2 \%$ observed for synonymous mutations. The signal for deleterious mutations was substantially reduced if TP53 was excluded (34.9\% of deleterious and $28.5 \%$ of other non-synonymous mutations had LOH). Thus, regions of $\mathrm{LOH}$ are slightly enriched for deleterious mutations, but not for other non-synonymous or silent mutations.

We then evaluated whether there was a difference in mutation frequency in CNN versus CNL regions of LOH on a case by case basis as above (Fig. 3). Excluding TP53, there were fewer mutations in regions of CNL$\mathrm{LOH}$ than would have been expected based on the overall percentage of the exome affected $(19.8 \%$ of mutations were in CNL-LOH regions, whereas $26.8 \%$ of the exome was affected by CNL-LOH, $\mathrm{p}<0.0001$, Binomial test). The difference was less striking when considering overtly deleterious mutations only $(24.6 \%$ vs $26.8 \%$, p = 0.09 , Binomial test). For CNN-LOH, the overall difference was small $(8.8 \%$ of mutations vs $8.7 \%$ of the exome affected by CNN-LOH, $\mathrm{p}=0.7$, Binomial test), however there were more deleterious mutations than 

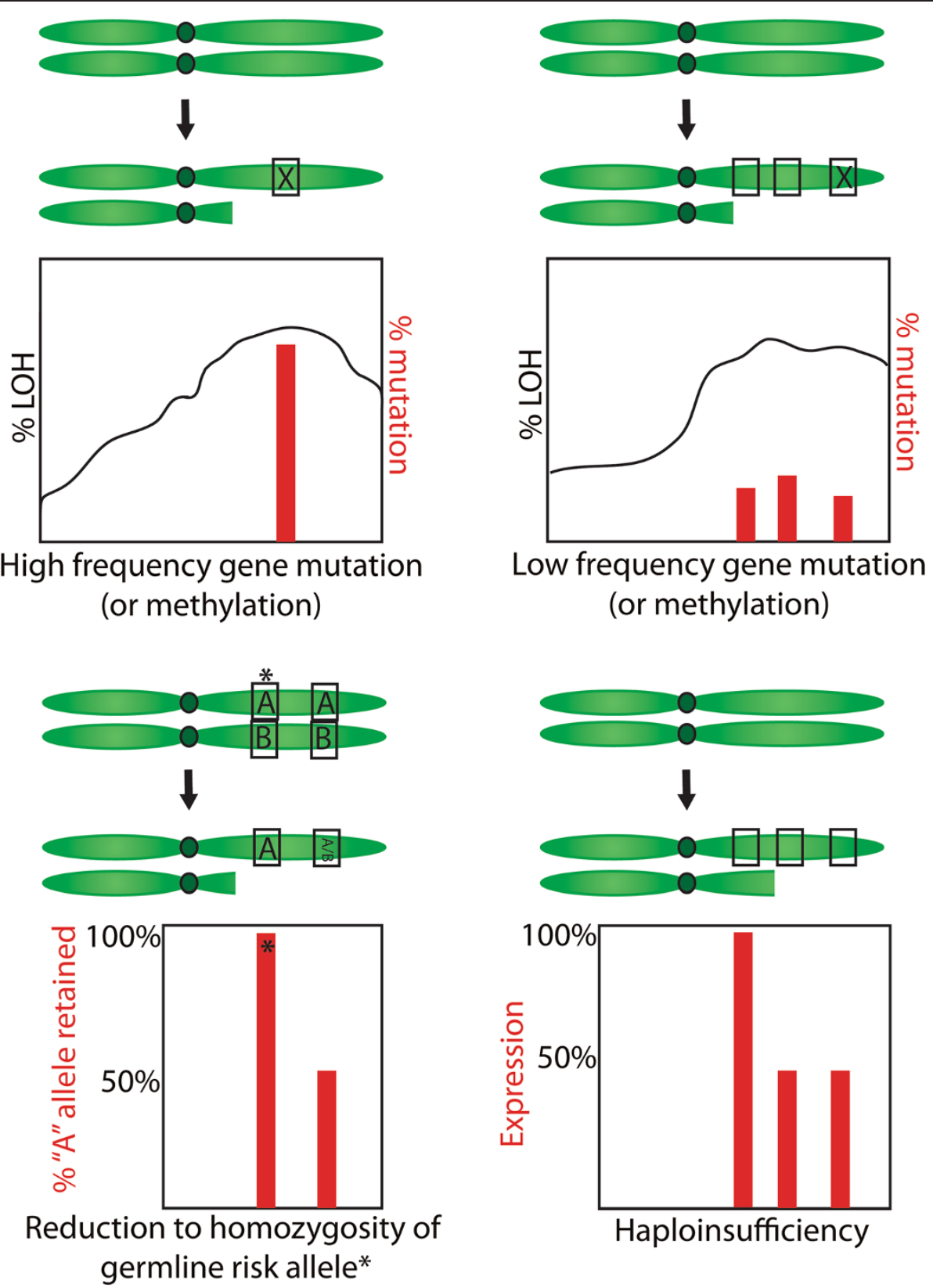

Fig. 1 Models of $\mathrm{LOH}$. Boxes = genes; " $X$ " = inactivating mutation; $A, B=$ alternative alleles of a single nucleotide polymorphism. In the top panels, the black line on the graph represents the overall frequency of $\mathrm{LOH}$ observed in tumour samples across the chromosome, while the red bars are the frequency of mutation in a particular gene. Thus, for the classic two-hit model, the frequency of mutation is similar to the frequency of LOH, while in the low frequency model, the frequency of $\mathrm{LOH}$ is higher than the mutation rate, because each sample is mutated in a different gene. In the bar graphs below, at left, the red bars represent the frequency of the A allele that is retained in samples with LOH at the locus; thus, the risk locus $\left(^{*}\right)$ has a higher proportion of the risk allele $(A)$ retained after $\mathrm{LOH}$ compared to a non-risk locus, where the $\mathrm{A}$ and $\mathrm{B}$ alleles are equally retained. At right, the graphs represents the average reduction in expression of a gene in samples with $L O H$, compared to samples without $L O H$; genes in $\mathrm{LOH}$ regions show a reduction in expression

expected ( $10.3 \%$ vs $8.7 \%, \mathrm{p}=0.05$, Binomial test). When TP53 was included, both total mutations (9.2 \%) and deleterious mutations (11.4\%) in CNN-LOH regions were increased. Silent mutations were the most likely to be underrepresented in CNL-LOH regions (17.2 \% vs $26.8 \%$ ).
It is possible, therefore, that mutations are seen less often in CNL-LOH regions simply as a consequence of decreased DNA dosage. The enrichment of deleterious mutations in $\mathrm{CNN}-\mathrm{LOH}$ regions, however, suggests the presence of positive selection for mutations in TSGs. 


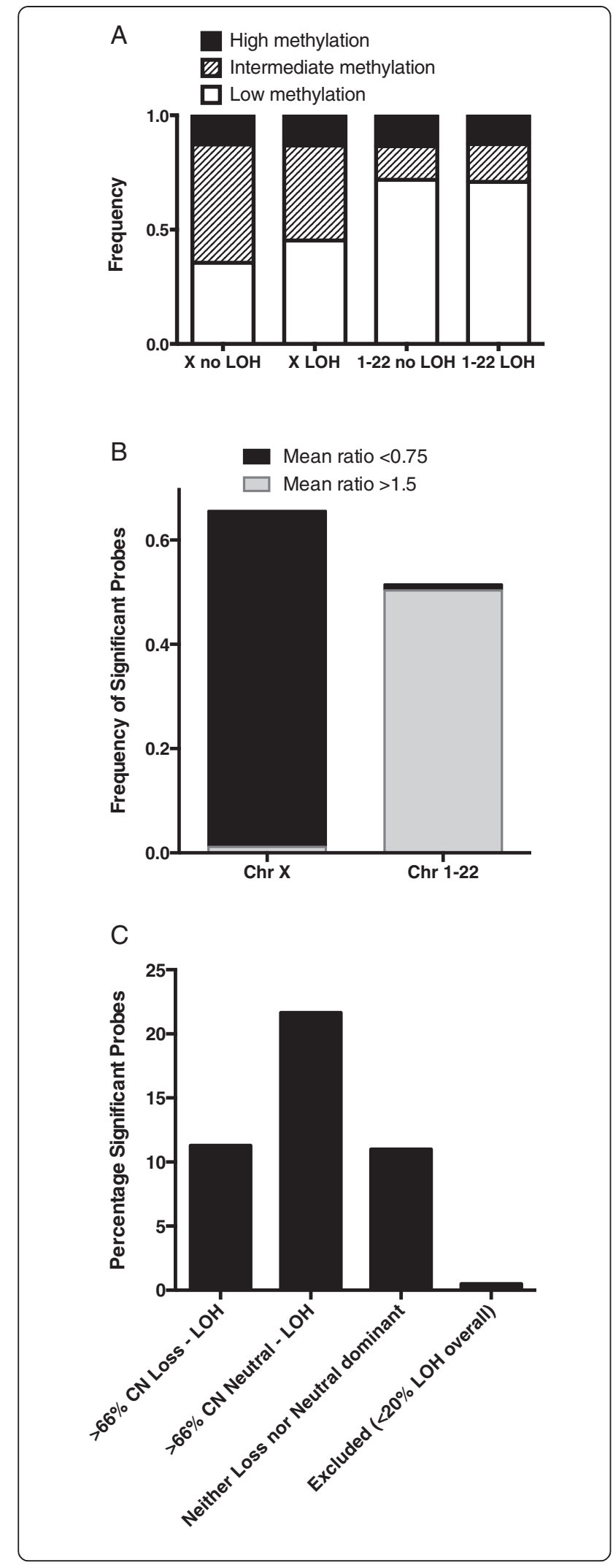

Fig. 2 Methylation. a Frequency of probes on the HM27 methylation array that have high (value $>0.75)$, intermediate $(0.25-0.75)$ and low $(<0.25)$ methylation associated with $\mathrm{LOH}$ in a sample, comparing all autosomes (no difference between $\mathrm{LOH}$ and no $\mathrm{LOH}$ ) and chromosome $\mathrm{X}$ (more low methylation probes in $\mathrm{LOH}$ regions). $\mathbf{b}$ Considering only probes that were significantly different between $\mathrm{LOH}$ and no $\mathrm{LOH}$, the frequency of significant probes where the mean methylation ratio $(\mathrm{LOH} / \mathrm{no} \mathrm{LOH})$ was increased $(>1.5$, higher in $\mathrm{LOH})$ or decreased $(<0.75$, higher in no $L O H)$. c Percentage of significant methylation probes that are located in regions where the majority $(>2 / 3)$ of $\mathrm{LOH}$ is either copy number loss, neutral or neither. Only regions with at least $20 \% \mathrm{LOH}$ were included

\section{Haplo-insufficiency: Single copy gene disruption}

We and others have shown that loss of a single gene copy can reduce gene expression [6,21]. A recent study showed that regions of copy number loss are enriched for tumour-suppressor genes [22], but that each gene might have a limited effect on its own. Chromosome complementation studies, where all or part of a chromosome is introduced into cell lines with $\mathrm{LOH}$ of that chromosome via microcell-mediated monochromosome transfer, have frequently been able to show reduction in tumorigenicity of the cell line thus complemented $[23,24]$, but have only rarely been able to implicate a single gene responsible $[25,26]$. Thus, haplo-insufficiency of multiple genes, each with a small effect, could contribute to the non-random pattern of $\mathrm{LOH}$ observed in ovarian cancer, especially for chromosomal regions that are weighted towards CNL-LOH such as $8 \mathrm{p}$ and $\mathrm{X}$, rather than $\mathrm{CNN}-\mathrm{LOH}$, such as 17 .

We previously observed a correlation between the percentage of genes under-expressed and the percentage of cases with CNL-LOH, as opposed to CNN-LOH, in a region-wise comparison of $\mathrm{LOH} v s$. no $\mathrm{LOH}$ [6]. In an analysis of TCGA data, we compared the expression of

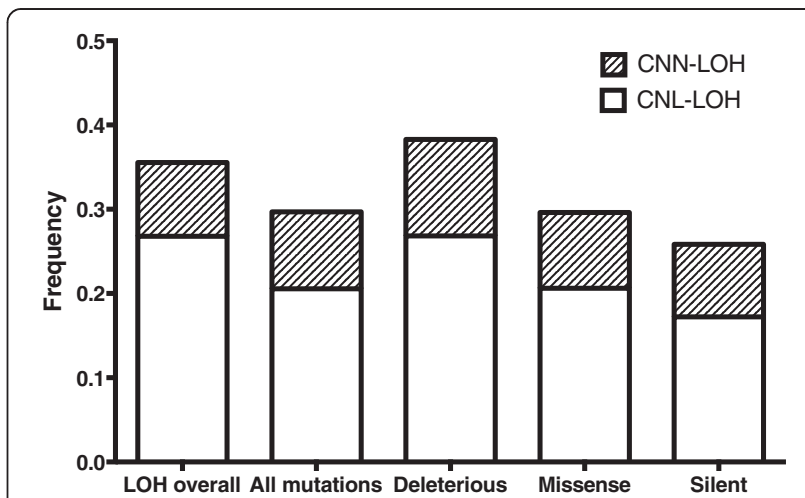

Fig. 3 Mutation load. Frequency of mutations of various types in copy number neutral and copy number loss regions, compared to the overall frequency of $\mathrm{LOH}$ across the exome ("LOH overall"). Deleterious mutations are enriched in CNN-LOH; all other mutations types are less frequent in copy number loss regions 
10,925 genes between cases with and without $\mathrm{LOH}$. 3,780 genes $(34.6 \%)$ were significantly differentially expressed (at a multiple testing p-value threshold of $\left.4.56 \times 10^{-6}\right)$, and all significant genes were underexpressed in samples with $\mathrm{LOH}$ compared to samples without $\mathrm{LOH}$. When comparing CNN-LOH to CNL-LOH in genes with at least $20 \%$ frequency of $\mathrm{LOH}$, only $1 / 163$ genes $(0.6 \%)$ at $\mathrm{CNN}-\mathrm{LOH}$ enriched loci were significantly differentially expressed, compared to 2701/5740 (47 \%) $\mathrm{CNL}-\mathrm{LOH}$ enriched genes $(\mathrm{p}<0.0001$, Chi-squared test, Fig. 4). This result supports the idea that chromosomal regions with CNL-LOH may contain genes where loss of a single copy results in reduced gene expression and a selective advantage to the cell. In contrast, chromosomal regions with little copy number loss may contain essential genes for which haplo-insufficiency is cell lethal.

5. Modified two-hit hypothesis: reduction to homozygosity of predisposition alleles

In familial cancer predisposition syndromes, it is common for the remaining wild-type allele to be lost by $\mathrm{LOH}$, for example $B R C A 1$ pathogenic variants are usually reduced to homozygosity in breast and ovarian carcinomas [3, 27]. However, common low-penetrance risk

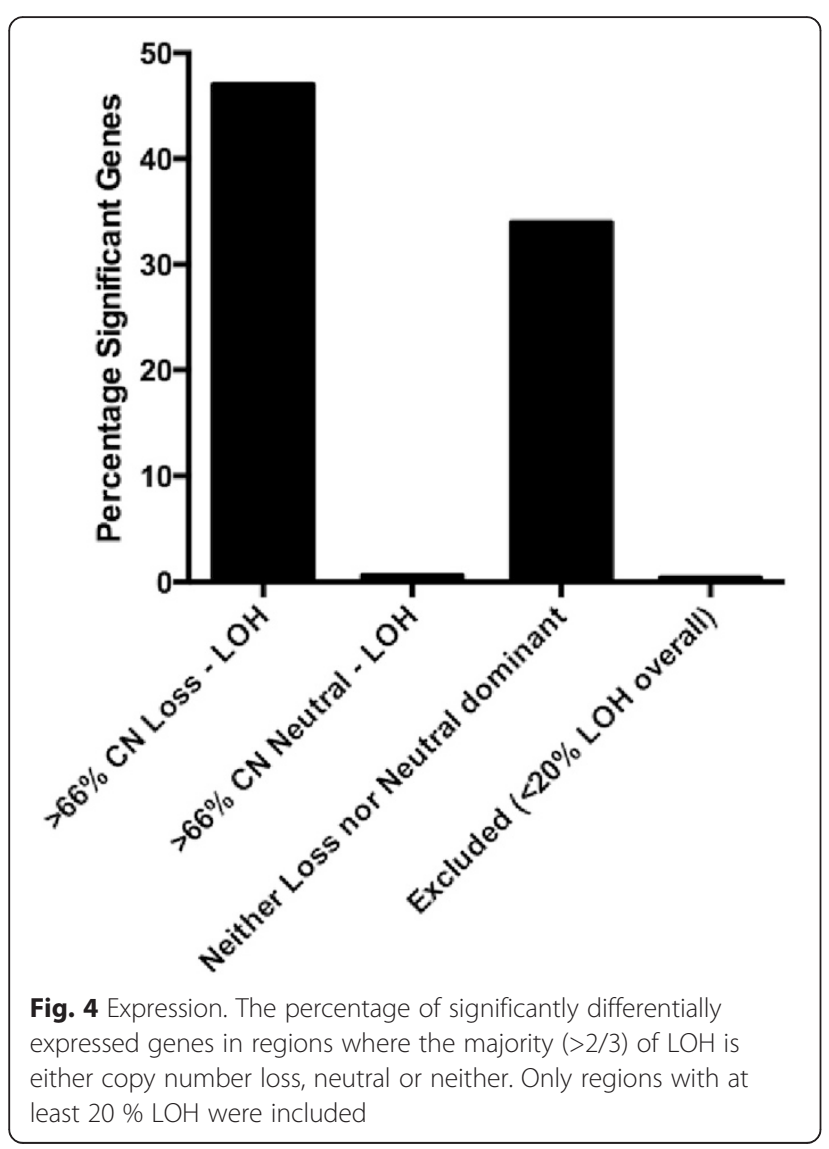

alleles could also be targeted by LOH leading to an enhancement of their cancer-promoting role. We assessed this using nine SNP loci identified in the iCOGs study $[28,29]$ as predisposing to all ovarian cancer types or high-grade serous ovarian cancer. Two of these SNPs were present on the Affymetrix SNP 6.0 array, the remainder were represented by SNPs in linkage disequilibrium $\left(r^{2}>0.7\right.$, from HapMap [30]). Where possible, up to four linked SNPs were evaluated.

For each SNP, we assessed whether cases were heterozygous in their normal DNA, and what proportion of these with $\mathrm{LOH}$ of the region were homozygous for the risk allele in the tumour DNA using TCGA and our own data $(\mathrm{n}=364)$. Interestingly, two SNPs at $10 \mathrm{p} 12$ linked to the risk allele rs1232180 were found to be significantly more likely to have lost the non-risk allele than the risk allele (Table 2). Some other SNPs linked to a risk allele also showed significantly non-random loss of the non-risk allele, but the data were not consistent across all SNPs examined at the locus (e.g. 17q21, 3q25 and 9p22). It is not clear whether these discrepancies could be due to technical variation in the SNP calling; alternative methods may be required to assess this possibility. The remainder of SNPs were not significant, however several are uncommon, limiting the power of the analysis. Thus, it is possible that some LOH may be selected for through the phenotypic effect of reduction to homozygosity of predisposition alleles.

\section{Conclusion}

The broader relevance of $\mathrm{LOH}$ in cancer has been debated for some time [5, 31] although many of the criticisms stemmed from technical issues that are being overcome by newer methodologies. Our initial assumption for this study was that we would detect high-frequency mutated genes in the minimal peak regions of $\mathrm{LOH}$ we had defined by $\mathrm{LOH}$ mapping using these newer methodologies; i.e. a classic two-hit model. However, the biology of LOH does not support this assumption and with large-scale tumour studies it is now possible to explore the many possibilities for the functional significance of this genetic event as summarised in Table 3. We suggest that the non-random patterns of $\mathrm{LOH}$ detected in cancer are a result of multiple different mechanisms operating to affect multiple genes, which may differ from tumour to tumour yet collectively play a role in the development of the tumorigenic phenotype. It is worth noting the differences in CNL-LOH versus CNN- LOH, with the latter appearing more relevant for selection of deleterious mutations and methylation, in contrast to global changes in gene expression. Identifying the specific driver genes targeted in a particular cancer remains a challenge given the multiple possible reasons for selection of an $\mathrm{LOH}$ event. 
Table 2 Ovarian cancer GWAS SNPs and LOH

\begin{tabular}{|c|c|c|c|c|c|c|c|c|c|c|c|c|c|c|}
\hline $\mathrm{SNP}^{\mathrm{a}}$ & Risk Allele $^{b}$ & Locus & het $^{c}$ & hom & AA & BB & $\mathrm{NC}$ & $\mathrm{N}$ & $\mathrm{CHI} \mathrm{sq}$ & Direction & $\% \mathrm{LOH}^{\mathrm{d}}$ & $\mathrm{N} L O H$ & Affy SNP & Rsquared \\
\hline rs1243180 & $\mathrm{NA}$ & 10p12 & & & & & & & & & & & & \\
\hline rs1243188 & minor/A & 10p12 & 112 & 183 & 22 & 6 & 41 & 364 & 0.0025 & yes & 0.20 & 28 & SNP_A-2024177 & 0.881 \\
\hline rs7098100 & minor/B & 10p12 & 132 & 181 & 1 & 9 & 41 & 364 & 0.0114 & yes & 0.07 & 10 & SNP_A-8636193 & 0.781 \\
\hline rs757210 & NA & $17 q 12$ & & & & & & & & & & & & \\
\hline rs11658063 & minor/B & $17 q 12$ & 23 & 148 & 44 & 44 & 105 & 364 & 1.0000 & . & 0.79 & 88 & SNP_A-8714923 & 0.704 \\
\hline rs9303542 & NA & $17 q 21$ & & & & & & & & & & & & \\
\hline rs4451990 & minor/B & $17 q 21$ & 20 & 197 & 49 & 61 & 37 & 364 & 0.2526 & & 0.85 & 110 & SNP_A-2282117 & \\
\hline rs12944592 & minor/B & $17 q 21$ & 24 & 198 & 54 & 60 & 28 & 364 & 0.5741 & & 0.83 & 114 & SNP_A-1836563 & 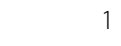 \\
\hline rs12452212 & minor/A & $17 q 21$ & 19 & 196 & 74 & 53 & 22 & 364 & 0.0624 & yes & 0.87 & 127 & SNP_A-2128564 & \\
\hline rs9894812 & minor/A & $17 q 21$ & 20 & 198 & 70 & 48 & 28 & 364 & 0.0428 & yes & 0.86 & 118 & SNP_A-2209606 & 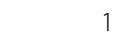 \\
\hline rs8170 & NA & 19p13 & & & & & & & & & & & & \\
\hline rs34084277 & minor/B & 19p13 & 80 & 260 & 9 & 8 & 7 & 364 & 0.8084 & . & 0.18 & 17 & SNP_A-1788674 & . \\
\hline rs2072590 & NA & $2 q 31$ & & & & & & & & & & & & \\
\hline rs711830 & minor/B & $2 q 31$ & 77 & 160 & 8 & 14 & 105 & 364 & 0.2008 & & 0.22 & 22 & SNP_A-8652216 & 0.965 \\
\hline rs7651446 & NA & $3 q 25$ & & & & & & & & & & & & \\
\hline rs344008 & minor/A & $3 q 25$ & 58 & 297 & 2 & 3 & 4 & 364 & 0.6547 & & 0.08 & 5 & SNP_A-8543714 & 0.85 \\
\hline rs2292336 & minor/B & $3 q 25$ & 48 & 299 & 2 & 5 & 10 & 364 & 0.2568 & . & 0.13 & 7 & SNP_A-8587822 & 0.85 \\
\hline rs17380639 & minor/A & $3 q 25$ & 28 & 320 & 11 & 3 & 2 & 364 & 0.0325 & yes & 0.33 & 14 & SNP_A-2078455 & 0.85 \\
\hline rs11782652 & minor/B & $8 q 21$ & 38 & 291 & 8 & 7 & 20 & 364 & 0.7963 & . & 0.28 & 15 & SNP_A-8702651 & \\
\hline rs10088218 & major/A & $8 q 24$ & 47 & 280 & 11 & 11 & 15 & 364 & 1.0000 & . & 0.32 & 22 & SNP_A-1801410 & \\
\hline rs1516974 & major/A & $8 q 24$ & 45 & 291 & 4 & 15 & 9 & 364 & 0.0116 & no & 0.30 & 19 & SNP_A-2088878 & \\
\hline rs3814113 & NA & $9 p 22$ & & & & & & & & & & & & \\
\hline rs7032221 & major/B & $9 p 22$ & 123 & 201 & 6 & 11 & 23 & 364 & 0.2253 & yes & 0.12 & 17 & SNP_A-8603886 & I \\
\hline rs10738467 & major/B & $9 p 22$ & 111 & 206 & 5 & 11 & 31 & 364 & 0.1336 & yes & 0.13 & 16 & SNP_A-8328297 & 0.892 \\
\hline rs10962668 & major/B & $9 p 22$ & 103 & 177 & 7 & 22 & 55 & 364 & 0.0053 & yes & 0.22 & 29 & SNP_A-4198891 & 0.794 \\
\hline
\end{tabular}

a SNPs in bold are those named in the GWAS iCOG publication [29] All others are linked as indicated by the R-squared value of >0.7. If the minor allele is the risk allele named, it is assumed that this will also be the case for the linked SNP

${ }^{\mathrm{b}}$ Minor = risk allele is the less frequent allele in the population. $\mathrm{A}, \mathrm{B}=$ risk allele corresponds to the " $\mathrm{A}$ " or " $\mathrm{B}$ " allele respectively in the Affymetrix array nomenclature. NA = not on Affymetrix SNP6 array

${ }^{c}$ Het $=$ Number of cases where germline and tumour are heterozygous, hom $=$ cases where germline is heterozygous, AA, BB = germline is heterozygous, tumour is homozygous for $\mathrm{A}$ or $\mathrm{B}$ respectively, $\mathrm{NC}=$ no call in either tumour or germline. $\mathrm{N}=$ total number

$\mathrm{d} \% \mathrm{LOH}$ is the number of individuals with loss of one allele divided by the total number of heterozygous individuals as measured at that SNP, i.e. not the overall $\%$ of $\mathrm{LOH}$ that could be determined from all cases using a wider genetic window. This may therefore include regions of extreme allelic imbalance (e.g. likely for $8 \mathrm{q} 24)$

Table 3 Summary of $\mathrm{LOH}$ - what is it good for?

\begin{tabular}{|c|c|c|c|c|c|}
\hline Hypothesis & Mechanism & Plausibility & Frequency & Impact & LOH type \\
\hline Classic two-hit hypothesis & $\begin{array}{l}\text { High frequency biallelic genetic inactivation of TSG } \\
\text { via mutation and } \mathrm{LOH} \text { or homozygous deletion }\end{array}$ & Strong & Rare & High & More CNN-LOH \\
\hline Modified two-hit hypothesis & $\begin{array}{l}\text { Reduction to homozygosity of predisposition } \\
\text { alleles }\end{array}$ & Low & Rare & Low & Unknown \\
\hline Epigenetic two-hit hypothesis & Biallelic inactivation through methylation and $\mathrm{LOH}$ & Moderate & Unknown & Moderate & More CNN-LOH \\
\hline Haplo-insufficiency & $\begin{array}{l}\text { Single copy gene disruption through copy number } \\
\text { loss }\end{array}$ & Strong & Common & Moderate & CNL-LOH \\
\hline Multi-gene biallelic inactivation & $\begin{array}{l}\text { Low frequency gene disruption through all of the } \\
\text { above mechanisms }\end{array}$ & Strong & Common & Unknown & Either \\
\hline
\end{tabular}




\section{Additional files}

Additional file 1: Table S1. Cohort information, Table S2. Regions of $\mathrm{LOH}$ analysed by targeted sequencing, Table $\mathbf{S 3}$. List of the genes in regions of $\mathrm{LOH}$ analysed by targeted sequencing. (DOCX $135 \mathrm{~kb}$ )

Additional file 2: Table S4. Excel file with all synonymous and nonsynonymous mutations identified by targeted sequencing. (XLSX $141 \mathrm{~kb})$

\section{Abbreviations}

AOCS: Australian Ovarian Cancer Study; LOH: Loss of heterozygosity; CNL-LOH: Copy number loss LOH; CNN-LOH: Copy number neutral $\mathrm{LOH}$ SNP: Single nucleotide polymorphism; TCGA: The Cancer Genome Atlas; TSG: Tumour suppressor gene.

\section{Competing interests}

The authors declare that they have no competing interests.

\section{Author contributions}

GLR conducted SNP array, mutation validation and all massively parallel sequencing experiments and analysed sequencing data; $M A D$ and $J L$ performed sequencing alignments and variant calling; DG performed bioinformatics analyses; SEB, DYHC and SMR extracted DNA and undertook Sanger sequencing validation; AOCS and DDLB contributed samples and data; RWT contributed to assay design and optimisation; IGC conceived of the study and participated in its design and coordination; and KLG conceived of the study, participated in its design and coordination, undertook bioinformatics analyses and drafted the manuscript. All authors read and approval the final manuscript.

\section{Author's information}

lan G. Campbell and Kylie L. Gorringe, co-senior authors.

\section{Acknowledgements}

We gratefully acknowledge the cooperation of the institutions in Australia participating in the Australian Ovarian Cancer Study (AOCS). We also acknowledge the contribution of the AOCS study nurses, research assistants and all clinical and scientific collaborators and would like to thank all of the women who participated in AOCS. The AOCS management group comprises David Bowtell (Peter MacCallum Cancer Centre, East Melbourne, Victoria, Australia), Georgia Chenevix-Trench (Queensland Institute of Medical Research, Brisbane, Queensland, Australia), Adele Green (Queensland Institute of Medical Research, Brisbane, Queensland, Australia), Penny Webb (Queensland Institute of Medical Research, Brisbane, Queensland, Australia), Anna deFazio (Westmead Institute for Cancer Research, Westmead Millennium Institute, Westmead, New South Wales, Australia ), Dorota Gertig (Victorian Cervical Cytology Registry, Carlton South, Victoria, Australia. The scientific and clinical collaborators are: ACT- R Stuart-Harris; NSW- F Kirsten, J Rutovitz, P Clingan, A Glasgow, A Proietto, S Braye, G Otton, J Shannon, T Bonaventura, I Stewart, S Begbie, M Friedlander, D Bell, S Baron-Hay, A Ferrier (dec.), G Gard, D Nevell, N Pavlakis, S Valmadre, B Young, C Camaris, R Crouch, L Edwards, N Hacker, D Marsden, G Robertson, P Beale, J Beith, J Carter, C Dalrymple, R Houghton, P Russell, L Anderson, M Links, J Grygiel, J Hill, A Brand, K Byth, R Jaworski, P Harnett, R Sharma, G Wain; QLD- D Purdie, D Whiteman, B Ward, D Papadimos, A Crandon, M Cummings, K Horwood. A Obermair, L Perrin, D Wyld, J Nicklin; SA- M Davy, MK Oehler, C Hall, T Dodd, T Healy, K Pittman, D Henderson, J Miller, J Pierdes, A Achan; TAS- P Blomfield, D Challis, R Mclntosh, A Parker; VIC- B Brown, R Rome, D Allen, P Grant, S Hyde, R Laurie M Robbie, D Healy, T Jobling, T Manolitsas, J McNealage, P Rogers, B Susil, E Sumithran, I Simpson, I Haviv, K Phillips, D Rischin, S Fox, D Johnson, S Lade, P Waring, M Loughrey, N O'Callaghan, B Murray, L Mileshkin, P Allan; V Billson, J Pyman, D Neesham, M Quinn, A Hamilton, O McNally, C Underhill, R Bell, LF Ng, R Blum, V Ganju; WA-I Hammond, A McCartney (dec.), C Stewart, Y Leung, M Buck, N Zeps (WARTN). Further information can be found at http://www.aocstudy.org. This work was supported by the National Health and Medical Research Council of Australia (NHMRC ID \#628773) and the Emer Casey Foundation.

\section{Author details}

${ }^{1}$ Cancer Genetics Laboratory, Peter MacCallum Cancer Centre, East Melbourne, Victoria, Australia. ${ }^{2}$ Centre for Cancer Research, Monash Institute of Medical Research, Monash University, Clayton, Victoria, Australia. ${ }^{3}$ Bioinformatics Core Facility, Peter MacCallum Cancer Centre, East Melbourne, Victoria, Australia. ${ }^{4}$ Bioinformatics and Cancer Genomics Laboratory, Peter MacCallum Cancer Centre, East Melbourne, Victoria, Australia. ${ }^{5}$ Sir Peter MacCallum Department of Oncology, University of Melbourne, Parkville, Victoria, Australia. ${ }^{6}$ Peter MacCallum Cancer Centre, East Melbourne, Victoria, Australia. ${ }^{7}$ Cancer Genomics and Genetics Laboratory, Peter MacCallum Cancer Centre, East Melbourne, Victoria, Australia. ${ }^{8}$ Molecular Genomics Core Facility, Peter MacCallum Cancer Centre, East Melbourne, Victoria, Australia. ${ }^{9}$ Department of Pathology, University of Melbourne, Parkville, Victoria, Australia.

Received: 16 February 2015 Accepted: 22 July 2015

Published online: 01 August 2015

\section{References}

1. Vogelstein B, Papadopoulos N, Velculescu VE, Zhou S, Diaz Jr LA, Kinzler KW. Cancer genome landscapes. Science. 2013;339(6127):1546-58. doi:10.1126/ science.1235122.

2. Cavenee WK, Dryja TP, Phillips RA, Benedict WF, Godbout R, Gallie BL, et al. Expression of recessive alleles by chromosomal mechanisms in retinoblastoma. Nature. 1983;305(5937):779-84

3. Merajver SD, Frank TS, Xu J, Pham TM, Calzone KA, Bennett-Baker P, et al. Germline BRCA1 mutations and loss of the wild-type allele in tumors from families with early onset breast and ovarian cancer. Clin Cancer Res. 1995;1(5):539-44.

4. Knudson Jr AG. Mutation and cancer: statistical study of retinoblastoma. Proc Natl Acad Sci U S A. 1971;68(4):820-3.

5. Tomlinson IP, Lambros MB, Roylance RR. Loss of heterozygosity analysis: practically and conceptually flawed? Genes Chromosomes Cancer. 2002;34(4):349-53. doi:10.1002/gcc.10085.

6. Gorringe KL, Ramakrishna M, Williams LH, Sridhar A, Boyle SE, Bearfoot JL, et al. Are there any more ovarian tumor suppressor genes? A new perspective using ultra high-resolution copy number and loss of heterozygosity analysis. Genes Chromosomes Cancer. 2009:48(10):931-42.

7. Davis SJ, Choong DY, Ramakrishna M, Ryland GL, Campbell IG, Gorringe KL. Analysis of the mitogen-activated protein kinase kinase 4 (MAP2K4) tumor suppressor gene in ovarian cancer. BMC Cancer. 2011;11:173. doi:10.1186/ 1471-2407-11-173.

8. Bryan EJ, Watson RH, Davis M, Hitchcock A, Foulkes WD, Campbell IG. Localization of an ovarian cancer tumor suppressor gene to a $0.5-\mathrm{cM}$ region between D22S284 and CYP2D, on chromosome 22q. Cancer Res. 1996;56(4):719-21.

9. Ryland GL, Bearfoot JL, Doyle MA, Boyle SE, Choong DY, Rowley SM, et al. MicroRNA genes and their target 3'-untranslated regions are infrequently somatically mutated in ovarian cancers. PLoS One. 2012;7(4):e35805. doi:10.1371/journal.pone.0035805.

10. Li H, Durbin R. Fast and accurate short read alignment with BurrowsWheeler transform. Bioinformatics. 2009;25(14):1754-60. doi:10.1093/ bioinformatics/btp324.

11. Novocraft Technologies. http://www.novocraft.com.

12. McKenna A, Hanna M, Banks E, Sivachenko A, Cibulskis K, Kernytsky A et al. The Genome Analysis Toolkit: a MapReduce framework for analyzing nextgeneration DNA sequencing data. Genome Res. 2010;20(9):1297-303. doi:10.1101/gr.107524.110

13. Albers CA, Lunter G, Macarthur DG, McVean G, Ouwehand WH, Durbin R. Dindel: Accurate indel calls from short-read data. Genome Res. 2010. doi:10.1101/gr.112326.110

14. Robinson JT, Thorvaldsdottir H, Winckler W, Guttman M, Lander ES, Getz G et al. Integrative genomics viewer. Nat Biotechnol. 2011;29(1):24-6. doi:10.1038/nbt.1754

15. Ramakrishna M, Williams LH, Boyle SE, Bearfoot JL, Sridhar A, Speed TP, et al. Identification of candidate growth promoting genes in ovarian cancer through integrated copy number and expression analysis. PLoS One. 2010;5(4), e9983. doi:10.1371/journal.pone.0009983.

16. Pleasance ED, Cheetham RK, Stephens PJ, McBride DJ, Humphray SJ, Greenman CD, et al. A comprehensive catalogue of somatic mutations from a human cancer genome. Nature. 2010;463(7278):191-6. doi:10.1038/ nature08658. 
17. Dees ND, Zhang Q, Kandoth C, Wendl MC, Schierding W, Koboldt DC, et al. MuSiC: identifying mutational significance in cancer genomes. Genome Res. 2012;22(8):1589-98. doi:10.1101/gr.134635.111.

18. Ahmed AA, Etemadmoghadam D, Temple J, Lynch AG, Riad M, Sharma R, et al. Driver mutations in TP53 are ubiquitous in high grade serous carcinoma of the ovary. J Pathol. 2010;221(1):49-56. doi:10.1002/path.2696.

19. McManus DT, Murphy M, Arthur K, Hamilton PW, Russell SE, Toner PG. p53 mutation, allele loss on chromosome 17p, and DNA content in ovarian carcinoma. J Pathol. 1996;179(2):177-82. doi:10.1002/(SICI)10969896(199606)179:2<177::AID-PATH561>3.0.CO;2-2.

20. Cancer Genome Atlas Research N. Integrated genomic analyses of ovarian carcinoma. Nature. 2011:474(7353):609-15. doi:10.1038/nature10166.

21. Pollack JR, Sorlie T, Perou CM, Rees CA, Jeffrey SS, Lonning PE, et al. Microarray analysis reveals a major direct role of DNA copy number alteration in the transcriptional program of human breast tumors. Proc Natl Acad Sci U S A. 2002;99(20):12963-8.

22. Davoli T, Xu AW, Mengwasser KE, Sack LM, Yoon JC, Park PJ, et al. Cumulative haploinsufficiency and triplosensitivity drive aneuploidy patterns and shape the cancer genome. Cell. 2013;155(4):948-62. doi:10.1016/ j.cell.2013.10.011.

23. Cody NA, Ouellet V, Manderson EN, Quinn MC, Filali-Mouhim A, Tellis P, et al. Transfer of chromosome 3 fragments suppresses tumorigenicity of an ovarian cancer cell line monoallelic for chromosome 3p. Oncogene. 2007;26(4):618-32. doi:10.1038/sj.onc.1209821.

24. Stronach EA, Sellar GC, Blenkiron C, Rabiasz GJ, Taylor KJ, Miller EP, et al. Identification of clinically relevant genes on chromosome 11 in a functional model of ovarian cancer tumor suppression. Cancer Res. 2003;63(24):8648-55.

25. England NL, Cuthbert AP, Trott DA, Jezzard S, Nobori T, Carson DA, et al. Identification of human tumour suppressor genes by monochromosome transfer: rapid growth-arrest response mapped to $9 \mathrm{p} 21$ is mediated solely by the cyclin-D-dependent kinase inhibitor gene, CDKN2A (p16INK4A). Carcinogenesis. 1996;17(8):1567-75.

26. Murakami Y. Functional cloning of a tumor suppressor gene, TSLC1, in human non-small cell lung cancer. Oncogene. 2002;21(45):6936-48. doi:10.1038/sj.onc.1205825.

27. Berchuck A, Heron KA, Carney ME, Lancaster JM, Fraser EG, Vinson VL, et al. Frequency of germline and somatic BRCA1 mutations in ovarian cancer. Clin Cancer Res. 1998;4(10):2433-7.

28. Earp MA, Kelemen LE, Magliocco AM, Swenerton KD, Chenevix-Trench G Australian Cancer S, et al. Genome-wide association study of subtype-specific epithelial ovarian cancer risk alleles using pooled DNA. Hum Genet. 2014;133(5):481-97. doi:10.1007/s00439-013-1383-3.

29. Pharoah PD, Tsai YY, Ramus SJ, Phelan CM, Goode EL, Lawrenson K, et al. GWAS meta-analysis and replication identifies three new susceptibility loci for ovarian cancer. Nat Genet. 2013;45(4):362-70. doi:10.1038/ng.2564.70e1-2.

30. International HapMap C. The International HapMap Project. Nature. 2003;426(6968):789-96. doi:10.1038/nature02168.

31. Presneau N, Manderson EN, Tonin PN. The quest for a tumor suppressor gene phenotype. Curr Mol Med. 2003;3(7):605-29.

32. Futreal PA, Coin L, Marshall M, Down T, Hubbard T, Wooster R, et al. A census of human cancer genes. Nat Rev Cancer. 2004;4(3):177-83. doi:10.1038/nrc1299.

33. The Cancer Genome Atlas Research Network. Integrated genomic analyses of ovarian carcinoma. Nature. 2011:474(7353):609-15. doi:10.1038/ nature10166

34. Cerami E, Gao J, Dogrusoz U, Gross BE, Sumer SO, Aksoy BA et al. The cBio Cancer Genomics Portal: An Open Platform for Exploring Multidimensional Cancer Genomics Data. Cancer Discov. 2012;2(5):401-4. doi:10.1158/21598290.CD-12-0095

\section{Submit your next manuscript to BioMed Central and take full advantage of:}

- Convenient online submission

- Thorough peer review

- No space constraints or color figure charges

- Immediate publication on acceptance

- Inclusion in PubMed, CAS, Scopus and Google Scholar

- Research which is freely available for redistribution 